\title{
Two protein tyrosine phosphatases, Ptp2 and Ptp3, modulate the subcellular localization of the Hog1 MAP kinase in yeast
}

\author{
Christopher P. Mattison and Irene M. Ota ${ }^{1}$ \\ Department of Chemistry and Biochemistry, University of Colorado, Boulder, Colorado 80309-0215 USA
}

The MAP kinase Hog1 transiently accumulates in the nucleus upon activation. Although Hog1 nuclear export correlates with its dephosphorylation, we find that dephosphorylation is not necessary for export.

Unexpectedly, a strain lacking the nuclear protein tyrosine phosphatase, Ptp2, showed decreased Hog1 nuclear retention, while a strain lacking the cytoplasmic Ptp3 showed prolonged Hog1 nuclear accumulation, consistent with Ptp2 being a nuclear tether for Hog1 and Ptp3 being a cytoplasmic anchor. In support of this result PTP2 overexpression sequestered Hog1 in the nucleus while PTP3 overexpression restricted Hog1 to the cytoplasm. Thus, Ptp2 and Ptp3 regulate Hog1 localization by binding Hog1.

[Key Words: Hog1; PTP; dephosphorylation; nuclear and cytoplasmic anchoring]

Received April 23, 1999; revised version accepted March 27, 2000.

Signal is transmitted to the nucleus through MAP kinase (MAPK) pathways via nuclear accumulation of MAPK (Chen et al. 1992; Gonzalez et al. 1993; Lenormand et al. 1993, 1998; Brunet et al. 1999|. For example, the stressactivated MAPKs Hog1 in Saccharomyces cerevisiae and Spcl in Schizosaccharomyces pombe transiently accumulate in the nucleus in response to osmotic stress (Ferrigno et al. 1998; Gaits et al. 1998; Reiser et al. 1999), and the vertebrate extracellular signal-regulated kinase (ERK) accumulates in response to growth factors (Traverse et al. 1992; Khokhlatchev et al. 1998; Lenormand et al. 1998; York et al. 1998). One requirement for nuclear accumulation is phosphorylation of the activation loop. Mutation of the activation loop Thr and/or Tyr residues prevents signal-induced nuclear accumulation of Hogl and Spc1 (Ferrigno et al. 1998; Gaits et al. 1998; Reiser et al. 1999) as does mutation of the ERK activation loop (Lenormand et al. 1993; Khokhlatchev et al. 1998). Although phosphorylation of the activation loop is required for signal-induced nuclear accumulation, MAPK activity is not required. Hog 1 and ERK mutated at the ATP-binding loop accumulate in the nucleus in response to signal (Gonzalez et al. 1993; Ferrigno et al. 1998; Khokhlatchev et al. 1998; Reiser et al. 1999).

Regulation of MAPK export from the nucleus is not as well understood. Nuclear export proteins, including the importin $\beta$ homolog, Xpol, and the small GTP-binding protein, Ran-Gsp1, have been implicated in Hog1 export

${ }^{1}$ Corresponding author.

E-MAIL Irene.Ota@colorado.edu; FAX (303) 492-3586.
(Ferrigno et al. 1998); however, it is not clear whether the phosphorylation state of Hogl regulates export. This event seems possible because dephosphorylation of the activation loop phosphotyrosine occurs simultaneously with export (Ferrigno et al. 1998; Reiser et al. 1999). Our results indicate, however, that Hog1-pY dephosphorylation by the nuclear-localized protein tyrosine phosphatase Ptp2 or the cytoplasmic protein tyrosine phosphatase Ptp3, or Hog1-pThr dephosphorylation by a type-2C Ser/Thr phosphatase, Ptc1, does not regulate its export from the nucleus. Instead, Ptp2 modulates Hog1 localization by acting as a nuclear anchor for Hog1 while Ptp3 acts as a cytoplasmic tether.

\section{Results}

Dephosphorylation of Hog1 is not required for nuclear export during adaptation

To examine the effect of Hog1 dephosphorylation on its localization, we produced a strain expressing Hog1-GFP from a low-copy plasmid in a hog1s background. To quantify the features of this process, we determined the percentage of cells having greater nuclear Hog1 relative to cytoplasmic Hogl before and during exposure to osmotic stress. As described previously, Hog1 transiently accumulated in the nucleus in response to stress and began redistribution to the cytoplasm between 10-20 min (Figs. 1 and 2) when the level of Hog1-pY began to decrease (Fig. 3).

To test whether protein tyrosine phosphatase-depen- 


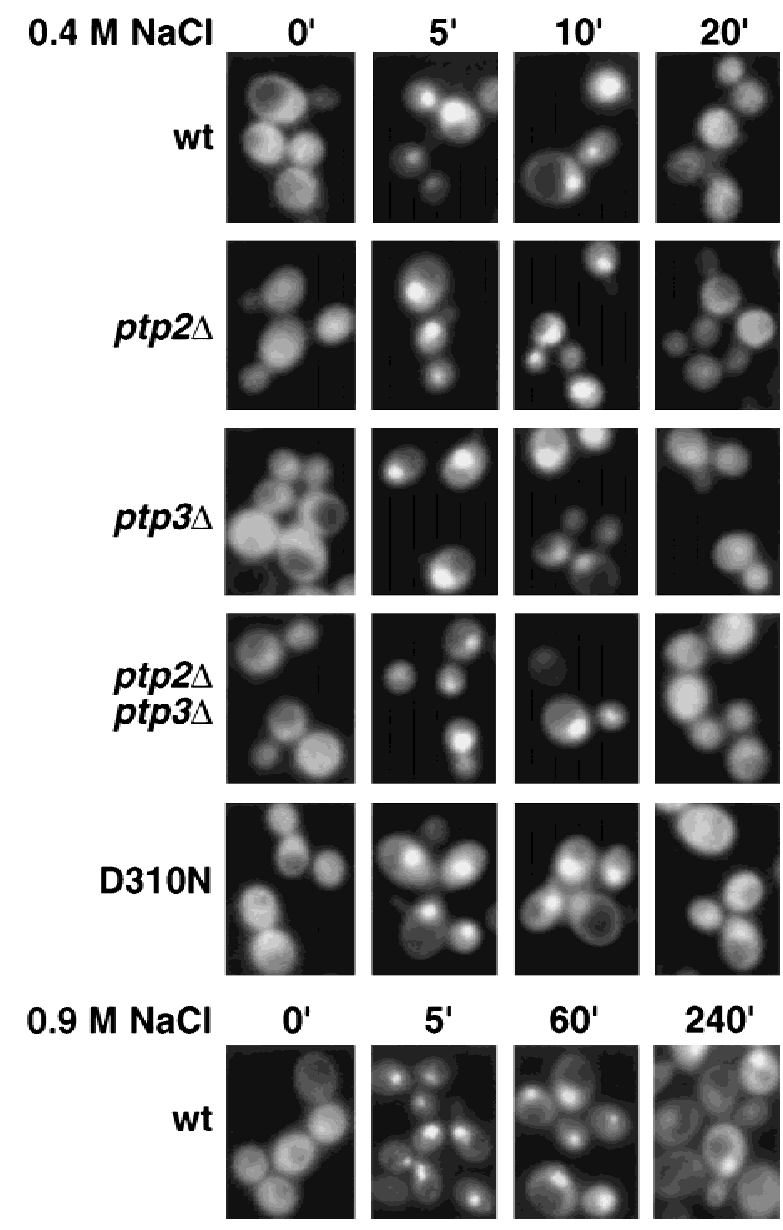

Figure 1. Redistribution of nuclear Hog1 to the cytoplasm does not require dephosphorylation of Hog1-pY. The subcellular localization of Hog1-GFP or Hog1-D310N-GFP in response to osmotic stress was examined in wild type or strains lacking PTP2, PTP3, or both PTP2 and PTP3. Exponentially growing cells were exposed to $0.4 \mathrm{M} \mathrm{NaCl}$ or $0.9 \mathrm{M} \mathrm{NaCl}$ for the indicated times. GFP and DAPI-stained nuclei were visualized by fluorescence microscopy.

dent dephosphorylation of Hog1-pY is required for its export from the nucleus, we examined Hog1 localization in strains lacking protein tyrosine phosphatases. Ptp2 and Ptp3 inactivate Hog1 by dephosphorylating Hog1$\mathrm{pY}$, with Ptp2 being the more potent negative regulator (Jacoby et al. 1997; Wurgler-Murphy et al. 1997). Ptp2 is predominantly nuclear while Ptp3 is cytoplasmic and appears to be excluded from the nucleus (Mattison et al. 1999). Therefore, deletion of PTP2 should delay Hog1 export if its dephosphorylation is necessary for export. Although the ptp2s strain poorly dephosphorylated Hog1-pY (Fig. 3), Hog1 redistribution to the cytoplasm was not delayed compared with wild type (Figs. 1 and 2A). For example, in the ptp2s mutant, the level of Hog1-pY remained high up to 40 min of osmotic stress; however, it was exported from the nucleus after $20 \mathrm{~min}$ (Figs. 1 and 2A). To examine further the effect of sustained Tyr phosphorylation, we examined Hog1 localization in the $p t p 2 \Delta$ tp $3 \Delta$ strain, which had an even greater defect dephosphorylating Hog1-pY (Fig. 3). Hog1 nuclear accumulation and redistribution to the cytoplasm in the ptp $2 \Delta$ ptp $3 \Delta$ strain was similar to wild type (Figs. 1 and 2A). These results indicate that Hog1-pY dephosphorylation is not required for export of Hogl from the nucleus.

Because Ptp2 and Ptp3 negatively regulate at least two other MAPK pathways (Zhan et al. 1997; Mattison et al. 1999|, deleting PTPs may indirectly affect Hogl localization by affecting other pathways. Therefore, we produced a mutant, Hog1-D310N, analogous to the sevenmaker mutant in the Drosophila rolled MAPK (Biggs et al. 1994; Bott et al. 1994; Brunner et al. 1994), that resists dephosphorylation in vivo (Fig. 3). Although Hog1-D310N was poorly dephosphorylated during adaptation, its subcellular localization was not appreciably altered from wild type (Figs. 1 and $2 \mathrm{~B}$ ), supporting further the idea that Hog1-pY dephosphorylation is not required for its export from the nucleus.

Hog1 activation and nuclear accumulation require phosphorylation of both Thr174 and Tyr176 in the activation loop (Schuller et al. 1994; Ferrigno et al. 1998; Reiser et al. 1999). To test whether Hog1-pThr174 dephosphorylation could be important for its export, we examined Hog1 localization in a strain lacking PTC1, encoding a type-2C Ser/Thr phosphatase. PTC1 was identified as a negative regulator of the HOG pathway by genetic means (Maeda et al. 1993, 1994), and more recently, we showed that Ptcl inactivates Hog1 in vivo and in vitro by specifically dephosphorylating pThr174 at the activation loop (J. Warmka and I. Ota, unpubl.). In the ptc1 $\Delta$ strain, Hog1 showed an increased basal level of activity, and was inactivated poorly as judged by Hog 1 kinase assays (J. Warmka and I. Ota, unpubl.); however, it accumulated in the nucleus and redistributed to the cytoplasm in a manner indistinguishable from wild type (Fig. 2C). These results indicate that dephosphorylation of neither Thr174 nor Tyr176 is necessary for export.

To test whether inhibiting dephosphorylation of both pThr174 and pTyr176 would prevent export, we examined Hog1 localization in a ptc1s ptp2s strain. This strain constitutively activates Hog1 and, therefore, exhibits a strong growth defect (Maeda et al. 1993; Jacoby et al. 1997). Despite this defect, the kinetics of Hog1 nuclear accumulation and redistribution to the cytoplasm were not significantly altered from wild type (Fig. 2C). These results suggest strongly that dephosphorylation of Hog1 at either p-Thr174 or p-Tyr176, and consequent inactivation of kinase activity is not required for redistribution of Hogl to the cytoplasm. These results also suggest that factors other than phosphorylation are required for nuclear accumulation of Hog1, because active Hog1, present in the $p t c 1 \Delta$ and $p t c 1 \Delta$ ptp $2 \Delta$ strains prior to stress, was not found accumulated in the nucleus (Fig. 2C).

Ptp2 and Ptp3 act as nuclear and cytoplasmic anchors for Hog1, respectively

Although the strain lacking nuclear Ptp2 did not slow 

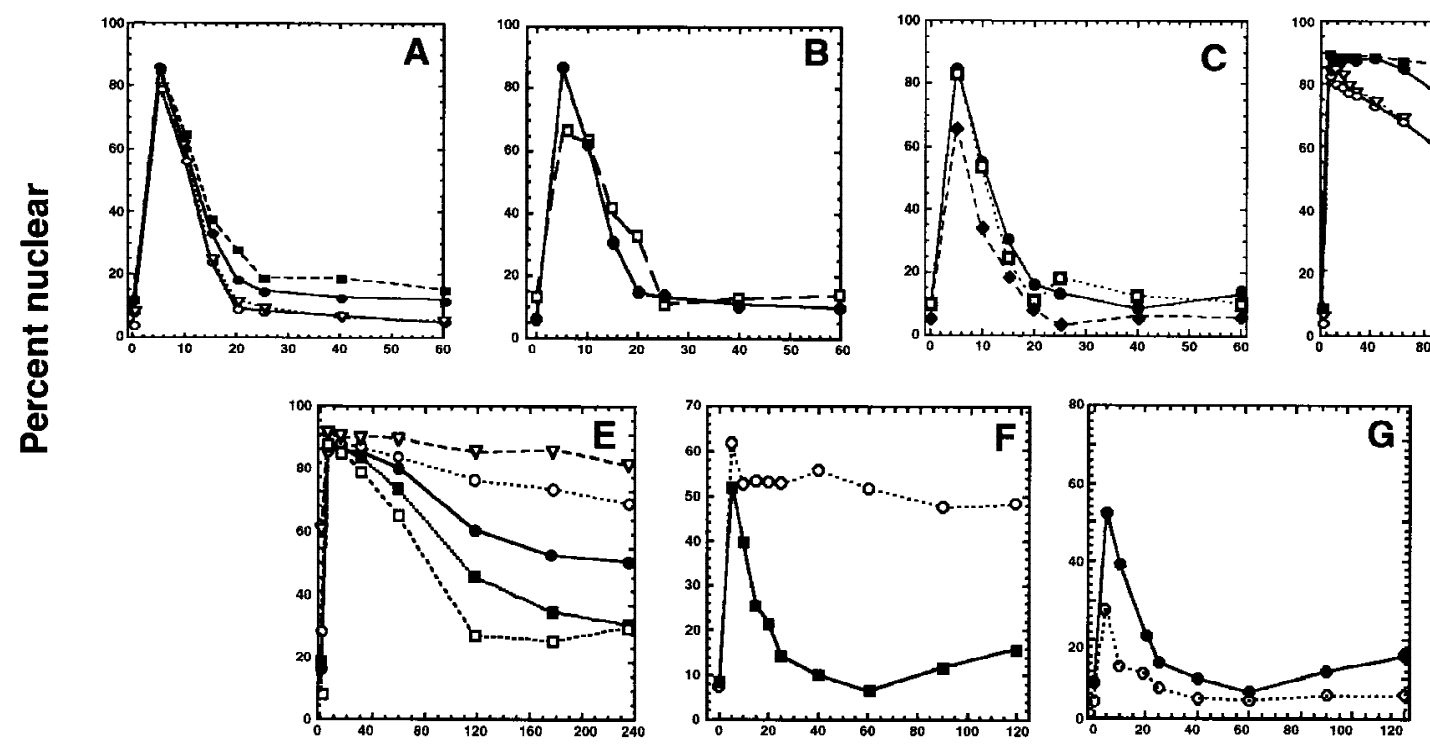

Time (min)

Figure 2. Hog1 dephosphorylation is not required for redistribution to the cytoplasm. Cultures expressing Hog1-GFP were exposed to $0.4 \mathrm{M} \mathrm{NaCl}$, unless otherwise noted, and the percentage of cells with greater nuclear relative to cytoplasmic accumulation determined. Approximately 100 cells were counted for each time point, and the average from at least two such experiments is shown.

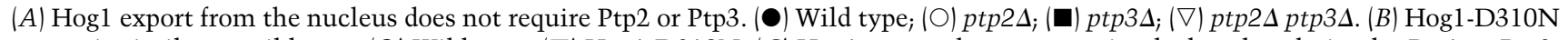
export is similar to wild type. (@) Wild type; ( $\square$ ) Hog1-D310N. (C) Hog1 export does not require dephosphorylation by Ptc1 or Ptp2. (๑) Wild-type; $(\square) p t c 1 \Delta_{;}(\bullet)$ ptc1s ptp2s. (D) Strains lacking PTP2 accumulate less Hog1 in the nucleus whereas strains lacking PTP3 accumulate more Hog 1 in the nucleus than wild type. Cells were exposed to $0.9 \mathrm{M} \mathrm{NaCl}$. Symbols are as in $A$. $(E)$ PTP catalytic activity is not required for anchoring Hog1. Wild-type or PTP-null strains expressing PTP catalytic mutants (Jacoby et al. 1997) were treated

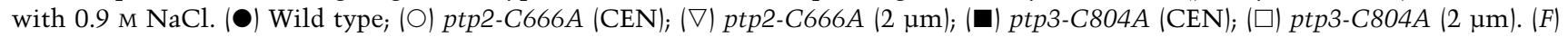
Hog1-K52M redistribution to the cytoplasm requires wild-type Hog1. HOG1 or hog1 $\Delta$ cells carrying pHOG1-K52M-GFP were exposed

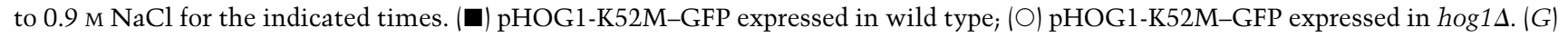
Hog1 kinase activity is not required to activate PTPs to redistribute Hog1-K52M to the cytoplasm. Exponentially growing wild type (-) or ptp2s (O) carrying pHOG1-K52M-GFP were exposed to $0.9 \mathrm{M} \mathrm{NaCl}$ for the indicated times.

Hog1 export from the nucleus, a slight decrease in the level of Hog1 nuclear accumulation was seen before and during osmotic stress (Fig. 2A). Conversely, the strain lacking cytoplasmic Ptp3 showed a slight increase in nuclear Hog1 (Fig. 2A). These results are consistent with Ptp2 acting as a nuclear tether for Hog1, and Ptp3 being a cytoplasmic anchor for Hog1. For example, the ptp2s strain should accumulate less Hogl in the nucleus than wild type if Ptp2 tethers Hog1 in the nucleus. Ptp2 and Ptp3 could function in this manner, as catalytically inactive mutants of Ptp2 and Ptp3 coprecipitate with Hog1 in yeast lysates (Jacoby et al. 1997; Wurgler-Murphy et al. 1997).

To test this idea further, we attempted to amplify the difference in Hog1-GFP localization between wild-type and PTP deletion strains by exposing cells to higher osmotic stress. Exposure to $0.9 \mathrm{M} \mathrm{NaCl}$ increased the duration of Hog 1 in the nucleus in both wild-type and $p t p \Delta$ strains (Fig.2, cf. A and D). Prolonged nuclear accumulation of $\mathrm{Hog} 1$ at $0.9 \mathrm{M} \mathrm{NaCl}$ is due to at least two factors. First, the level of Hog1-pY remained high for up to $4 \mathrm{hr}$ at $0.9 \mathrm{M} \mathrm{NaCl}$ compared with $15 \mathrm{~min}$ at $0.4 \mathrm{M} \mathrm{NaCl}$ in wild type (Fig. 3), suggesting that upstream components of the HOG pathway remain activated for a longer period of time at $0.9 \mathrm{M} \mathrm{NaCl}$. Second, more Hog1 was activated at $0.9 \mathrm{M} \mathrm{NaCl}$, as Hogl kinase activity was $\sim 1.5$-fold higher at $0.9 \mathrm{M} \mathrm{NaCl}$ compared with $0.4 \mathrm{M} \mathrm{NaCl}$ (data not

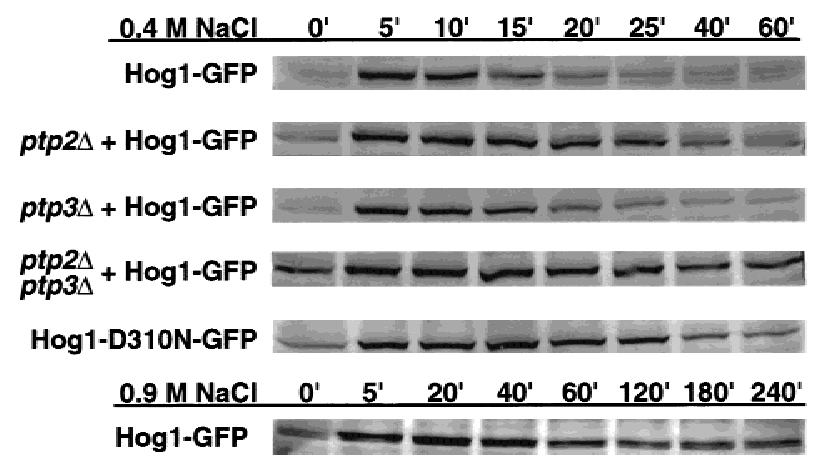

Figure 3. The level of Hog1-pY increases in $p t p \Delta$ strains and HOG1 mutants. Hog1-pY in hog1s strains wild type for PTPs or lacking PTPs was examined in exponentially growing cells carrying pHOG1-GFP (rows 1-4). The level of Hog1-D310N-pY was also examined in a hog $1 \Delta$ strain. Cells were exposed to 0.4 or 0.9 $\mathrm{M} \mathrm{NaCl}$, harvested at the indicated times, and lysates analyzed by SDS-PAGE and immunoblotting with anti-phosphotyrosine antibody. 
shown). Because dephosphorylation of Hog1 is not necessary for its export from the nucleus, we believe that the sustained nuclear accumulation of Hog1 at $0.9 \mathrm{M}$ $\mathrm{NaCl}$ could be due to re-import of phosphorylated Hog1. Under these conditions, the $p t p 2 \Delta$ strain retained significantly less Hog 1 in the nucleus than wild type, while the ptp3 $\Delta$ strain showed increased nuclear retention of Hog1 (Fig. 2D).

To test whether Ptp2 sequestration of Hog1 in the nucleus and Ptp3 tethering of Hog1 to the cytoplasm was a result of PTPs binding Hog1, or their catalytic activity, Hog1 localization was examined in ptp-null strains expressing catalytically inactive PTPs. Ptp2C666A shifted Hog 1 to the nucleus, while Ptp3C804A shifted Hog1 to the cytoplasm (Fig. 2E). Expression of these mutants from multicopy plasmids exacerbated these effects (Fig. $2 \mathrm{E})$. These results support strongly the idea that Ptp2 is a nuclear anchor whereas Ptp3 is a cytoplasmic tether for Hog 1 .

If PTPs function as anchors for Hog1, then their overexpression from the strong $G A L$ promoter might sequester Hog1 in the absence of signal. Indeed, PTP2 overexpression segregated the majority of Hog1 to the nucleus

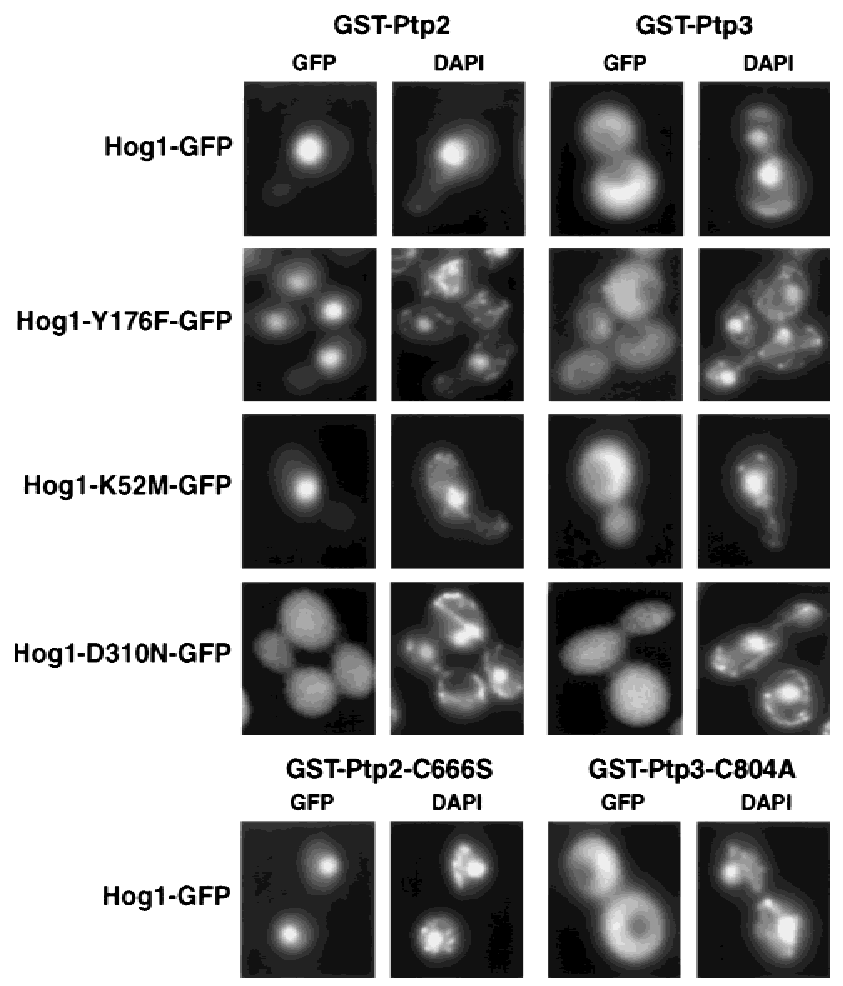

Figure 4. Overexpression of PTPs tethers Hog1 in different subcellular compartments. The subcellular localization of wildtype and mutant Hog1 proteins fused to GFP was examined in hog1A strains overexpressing PTP2 or PTP3 or their mutant counterparts, ptp2-C666S and ptp3-C804A. The Hog1 plasmids were pHOG1-GFP, pHOG-Y176F-GFP, pHOG1-K52M-GFP, and pHOG1-D310N-GFP. The PTP-overexpressing plasmids were pKT-PTP2, pKT-PTP3, pGST-PTP2-C666S, and pGSTPTP3-C804A. GFP and DAPI-stained nuclei were visualized by fluorescence microscopy.

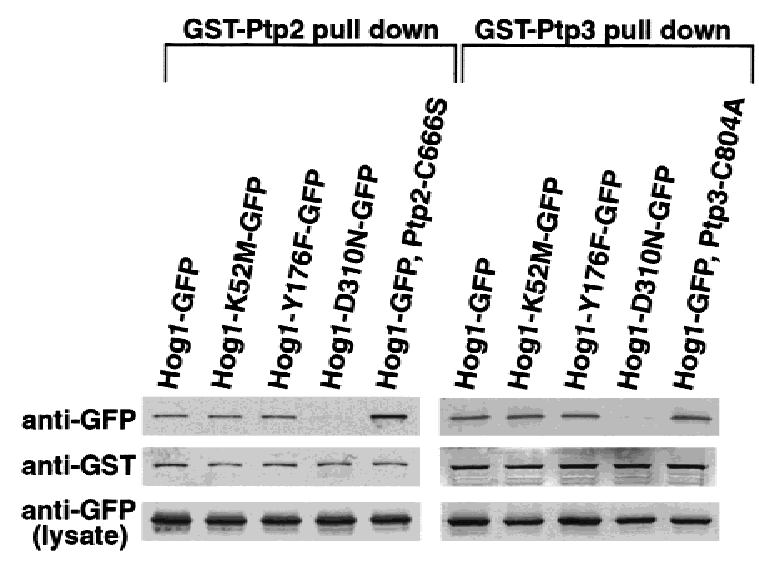

Figure 5. PTPs physically associate with Hog1. GST fusions to wild-type and mutant PTPs (Ptp2-C666S and Ptp3-C804A), were coexpressed with wild type and mutant Hog1-GFP fusions in yeast. GST fusion proteins were isolated using glutathioneSepharose and associated Hogl examined by SDS-PAGE and immunoblotting with anti-GST and anti-GFP antibodies as indicated.

while PTP3 overexpression sequestered Hog1 in the cytoplasm (Fig. 4 ). Colocalization was mediated by complex formation as wild-type PTPs coprecipitated with Hog1 (Fig. 5). Next, we examined the requirements for PTP-induced Hog1 compartmentalization. First, PTP catalytic activity was not required because overexpression of Ptp2-C666S sequestered Hog1 in the nucleus, whereas overexpression of Ptp3-C804A, localized Hog1 to the cytoplasm (Fig. 4). Second, neither Hog1 Tyr phosphorylation nor Hog1 activity was required for PTP tethering, as Hog1-Y176F and Hog1-K52M were concentrated in the nucleus by PTP2 overexpression (Fig. 4) and sequestered in the cytoplasm by PTP3 overexpression (Fig. 4). Colocalization was due to complex formation, as mutant PTP proteins coprecipitated with wild-type Hog1 as described previously (Fig. 5; Jacoby et al. 1997; Wurgler-Murphy et al. 1997) and mutant Hog1 coprecipitated with wild-type PTPs (Fig. 5). One mutant protein, Hog1-D310N, was not sequestered by overexpression of PTPs (Fig. 4) and bound poorly to PTPs (Fig. 5). Therefore, PTPs regulate Hogl localization by formation of tight complexes.

Activation of nuclear Ptp2 is not required for export of kinase-inactive Hog1

One factor important for export of Hogl from the nucleus is its kinase activity. A kinase-inactive mutant, Hog1-K52M, is retained in the nucleus following exposure to $0.4 \mathrm{M} \mathrm{NaCl}$ (Ferrigno et al. 1998; Reiser et al. 1999) and $0.9 \mathrm{M} \mathrm{NaCl}$ (Fig. 2F). Because osmotic stress increases the level of PTP2 and PTP3 mRNAs in a Hog1dependent manner (Jacoby et al. 1997; Wurgler-Murphy et al. 1997), it has been suggested that Hog1-K52M is retained in the nucleus because of its inability to activate PTPs. In support of this, Hog1-K52M-pY was de- 
phosphorylated poorly after exposure to $0.4 \mathrm{M} \mathrm{NaCl}$ (Wurgler-Murphy et al. 1997; Ferrigno et al. 1998) and 0.9 $\mathrm{M} \mathrm{NaCl}$ (data not shown). Furthermore, coexpression of wild-type HOG1 with Hog1-K52M-GFP, allows Hog1K52M-GFP to be dephosphorylated and exported from the nucleus at $0.4 \mathrm{M} \mathrm{NaCl}$ (Ferrigno et al. 1998; Reiser et al. 1999) and $0.9 \mathrm{M} \mathrm{NaCl}$ (Fig. 2F). These results suggest that Hog $1-\mathrm{K} 52 \mathrm{M}$ is retained in the nucleus because of its inability to be dephosphorylated by PTPs. Our results with wild-type Hog1, however, predicted that PTP dephosphorylation of Hog1-K52M is not required for export. Therefore, we examined Hog1-K52M-GFP export in a HOG1 PTP2 strain, and a HOG1 ptp2s strain. If Hog1-K52M-GFP is rapidly exported in the HOG1 strain because Ptp2 is activated, then Hog1-K52M export should be retarded when PTP2 is deleted. In the HOG1 ptp2s strain, Hog1-K52M-GFP did not accumulate in the nucleus as well as wild type; however, it redistributed to the cytoplasm in a manner similar to the HOG1 PTP2 strain (Fig. 2G). Thus, the rapid redistribution of Hogl-K52M to the cytoplasm in the HOG1 strain is likely due to factors other than Ptp2-mediated dephosphorylation.

\section{Discussion}

The subcellular localization of MAPKs is regulated by their phosphorylation. MAPK nuclear import requires phosphorylation of the activation loop Thr and Tyr residues. Although dephosphorylation of Hog1 is correlated with its export from the nucleus, we show that its dephosphorylation at pTyr or pThr in the activation loop is not required for export. Dephosphorylation may not regulate the export of other MAPKs. For example, the protein tyrosine phosphatases that inactivate the $S$. pombe stress-activated MAPK, Spc1, are found in the cytoplasm, suggesting that Spc1-pY dephosphorylation is not necessary for export (Gaits and Russell 1999).

One factor critical for Hogl export from the nucleus is its kinase activity (Ferrigno et al. 1998; Reiser et al. 1999). Here, we show that dephosphorylation of kinaseinactive Hog 1 is not necessary for export, suggesting that kinase activity is required to up-regulate or activate proteins other than phosphatases to allow export. For example, export of the vertebrate stress-activated MAPK, p38, from the nucleus is dependent on phosphorylation of its substrate, MAPKAP kinase-2 (Ben-Levy et al. 1998). An alternative explanation for the extended nuclear localization of Hog1-K52M, however, is that it binds nuclear substrates with greater affinity, and thereby prolongs nuclear accumulation. For example, similar mutations in MEK and MAPK bind their substrates with higher affinity (Zhou et al. 1995; Han et al. 1997).

A second factor that regulates MAPK localization is interaction with proteins in the cytoplasm and the nucleus. For example, MEK is proposed to be a cytoplasmic anchor for MAPK, tethering it in the cytoplasm prior to activation and releasing it upon activation to accumulate in the nucleus (Fukuda et al. 1997). In yeast stress- response pathways, MEK may not function in this manner. Deletion of MEK does not lead to nuclear accumulation of Hog1 (Reiser et al. 1999; C. Mattison and I. Ota, unpubl.) or the S. pombe Spc1 (Gaits et al. 1998). Nevertheless, cytoplasmic anchoring is important for Hog1 localization, as overexpression of Hog 1 in the absence of osmotic stress, or overexpression of the activation loop mutant, Hog1-T174A,Y176A, leads to nuclear accumulation (C. Mattison and I. Ota, unpubl.). Because Hog1 is more abundant that Pbs2 (C. Mattison and I. Ota, unpubl.), other cytoplasmic proteins are likely involved in tethering Hogl.

In this work, we identify Ptp3 as one such cytoplasmic tether for Hog1. Deletion of PTP3 elevated the level of Hog1 in the nucleus prior to and following osmotic stress. We find that Ptp3 is predominantly cytoplasmic and that its localization does not change in response to osmotic stress, consistent with it being a cytoplasmic tether (Mattison et al. 1999). Because there is less Ptp3 protein than Hog1 (C. Mattison and I. Ota, unpubl.), other proteins are also likely to anchor Hogl in the cytoplasm. Cytoplasmic phosphatases in other MAPK pathways may also function as tethers. For example, overexpression of the catalytically inactive dual-specificity phosphatase, MKP-3, or the protein tyrosine phosphatase, PTP-SL, has been shown to constrain ERK to the cytoplasm and prevent signaling (Brunet et al. 1999; Zuniga et al.).

Nuclear retention factors are also important in regulating MAPK signaling. In $S$. pombe, nuclear accumulation of Spc1 requires the presence of its nuclear-localized substrate, the transcriptional activator, Atf1 (Gaits et al. 1998; Watanabe and Yanamoto 1996). In this work, we show that a protein phosphatase, Ptp2, can also affect the localization of Hog1. Consistent with Ptp2 being a nuclear tether, it is concentrated in the nucleus, and its localization does not change upon osmotic stress (Mattison et al. 1999). Because deletion of PTP2 reduced but did not inhibit Hog1 nuclear accumulation, other nuclear proteins may also function as anchors for Hog1. For example, deletion of MSN2 and MSN4, general stress transcription factors, also inhibit maximal nuclear accumulation of Hog1 (Reiser et al. 1999).

Therefore, Ptp2 and Ptp3 have more than one role in regulating the HOG pathway. One is to inactivate Hog1 through dephosphorylation, while the second is to modulate Hog1 subcellular localization through binding interactions. These functions may be separated into different domains in the PTPs, which have a noncatalytic amino-terminal domain and a catalytic carboxy-terminal domain (Ota and Varshavsky 1992; Jacoby et al. 1997). PTP-Hog1 compartmentalization does not require PTP activity, Hog1-Tyr phosphorylation, or Hog1 kinase activity. Therefore, PTP-Hogl binding interactions could occur at sites separate from the PTP catalytic domain and the Hogl activation loop. Indeed, the isolated amino-terminal domain of PTPs bind Hog1 (C. Mattison, S. Spencer, and I. Ota, unpubl.), and the Hogl mutation, Hog1-D310N, which shows reduced binding to PTPs, is found on the face opposite Tyr176, suggesting that re- 
gions other than the activation loop can interact with PTPs. Therefore, binding between PTPs and Hogl could regulate signaling independent of catalytic activity. In support of this, overexpression of the catalytically inactive mutant, Ptp2-C666S, inactivates the HOG pathway (Jacoby et al. 1997; Wurgler-Murphy et al. 1997). Further study of the interactions with PTPs that modulate Hog1 localization are underway.

\section{Materials and methods}

Strains, media, and genetic techniques

Hog1-GFP was visualized in the hog1 $:: T R P 1$ strain, IMY100

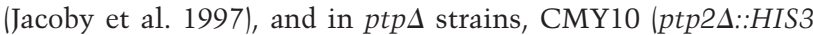
hog1 $1:: T R P 1)$, CMY11 (ptp3s::TRP1 hog1 $:: T R P 1)$, and CMY12

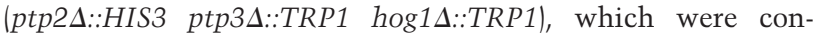
structed by crossing the previously described $p t p \Delta$ strains (Jacoby et al. 1997) to IMY100. Hog1-GFP was also visualized in

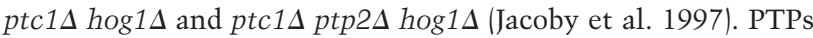
were overexpressed from the plasmids, pKT-Ptp2, pKTPtp2C666A, pKT-Ptp3, and pKT-Ptp3C804A (Jacoby et al. 1997) in a hog1s strain, IMY200, derived from 334 (Hovland et al. 1989). IMY200 was produced by transforming the 334 strain with the hog1D::hisG-URA3-hisG allele $\left(\mathrm{O}^{\prime}\right.$ Rourke and Herskowitz 1998) and growing on media containing 5-fluoroorotic acid (5-FOA; American Biorganics, Inc., Swampscott, MA) to isolate the $\operatorname{hog} 1 \Delta::$ his $G$ strain. Yeast transformations were performed according to Dohmen et al. (1991). Media to culture yeast were produced as described by Sherman et al. (1986).

\section{Plasmids}

Plasmids expressing wild-type and mutant Hog1-GFP were constructed as follows. A NotI site was substituted for the HOG1 stop codon by use of PCR and ligated to a 750-bp NotI fragment containing GFP (J. Hirsch, Columbia University, NY) in the CEN-based plasmid, YCplac111 (Gietz and Sugino 1988). The resulting pHOG1-GFP complemented the osmotic stress sensitivity of the hog1s strain, IMY100 (Brewster et al. 1993; Jacoby et al. 1997). The HOG1-Y176F allele was produced by PCR with the oligonucleotide, 5'-AAGAATTCAAGACCCTCAAATGACAGGCTTTGTTTCCACTAG-3', where the mutations are underlined. The HOG1-K52M allele was produced by PCR using the mutagenic oligonucleotide, 5'-CATCTCAGCCAGTTGCCATTATGAAAATCATG-3', containing the underlined mutations. A 500-bp EcoRI fragment containing the K52M mutation was substituted for the wild-type EcoRI fragment in a plasmid bearing the wild-type HOG1 gene. The HOG1-D310N allele was produced by site-directed mutagenesis of a 911-bp PstI fragment of the HOG1 ORF by use of the oligonucleotide, 5'-CGGCTACTGGTTCATTCGTTGGATCGTGG-3', containing the underlined mutations. This fragment was substituted for the wild-type PstI fragment in HOG1.

\section{Immunoblot analysis}

The level of Hog1-pY was monitored by immunoblotting. Cultures were grown to exponential phase at $30^{\circ} \mathrm{C}$ in synthetic media and were untreated or exposed to osmotic stress by the addition of $\mathrm{NaCl}$ to a final concentration of $0.4 \mathrm{M} \mathrm{NaCl}$ or $0.9 \mathrm{M}$ $\mathrm{NaCl}$. Approximately $7.5 \times 10^{7}$ cells were harvested by centrifugation, resuspended in $40 \mu \mathrm{l}$ of $90 \mathrm{mM}$ Na-HEPES (pH 7.5), 2\% SDS, and heated at $100^{\circ} \mathrm{C}$ for $3 \mathrm{~min}$ (Mattison et al. 1999). To this mixture, $40 \mu \mathrm{l}$ of ice-cold lysis buffer, $50 \mathrm{~mm}$ sodium
HEPES (pH 7.5), 5 mM EDTA, 150 mm NaCl, 1\% Triten-X 100, with protease inhibitors (leupeptin, pepstatin A, antipain, aprotinin, and chymostatin each at $20 \mu \mathrm{g} / \mathrm{ml}$, and $35 \mu \mathrm{g} / \mathrm{ml}$ PMSF), and protein phosphatase inhibitors, $1 \mathrm{~mm}$ sodium orthovanadate and $40 \mathrm{~mm} \beta$-glycerophosphate was added. Immunoblotting was performed as described previously (Jacoby et al. 1997) using PY-99 (Santa Cruz Biotechnology) and anti-GFP antibody generously provided by C. Zuker (UCSD). Immunoreactivity was visualized using alkaline phosphatase conjugated to antimouse antibody and 5-bromo-4-chloro-3-indolyl phosphate and nitro-blue tetrazolium.

\section{Fluorescence microscopy}

Strains expressing GFP-fusion proteins were grown to $\sim 1$ unit $\left(\mathrm{A}_{600}\right)$ in synthetic medium lacking leucine to select for plasmids. To visualize the nucleus, cultures were diluted to 0.50 units $\left(\mathrm{A}_{600}\right)$, 4',6-diamidino-2-phenylindole (DAPI) was added to a concentration of $2.5 \mu \mathrm{g} / \mathrm{ml}$, and the cultures grown under low light conditions for $30 \mathrm{~min}$ at $30^{\circ} \mathrm{C}$. Cultures were untreated or exposed to $0.4 \mathrm{M} \mathrm{NaCl}$ or $0.9 \mathrm{M} \mathrm{NaCl}$ for various times and the cells adsorbed to polylysine-coated slides (Polysciences, Inc., Warrington, PA). Fluorescence images were captured using a Zeiss Axioplan fluorescence microscope with a Photometrics Sensys digital CCD camera system. Images were processed using IP-LAB Spectrum software. Photographed cells were scored by eye as having nuclear Hog 1 accumulation if the nuclear GFP signal was more intense than the cytoplasmic signal.

\section{Coprecipitation assay}

Yeast expressing wild type or mutant GST-PTP fusions, and wild-type or mutant Hog1-GFP fusions were grown to $\sim 1$ unit (A600nm) in selective medium and harvested by centrifugation. Cells were lysed by glass beading in ice-cold lysis buffer, $50 \mathrm{~mm}$ Tris- $\mathrm{HCl}(\mathrm{pH} 7.5), 50 \mathrm{~mm} \mathrm{NaCl}, 5 \mathrm{~mm} \mathrm{MgCl}_{2}, 0.2 \%$ Triton $\mathrm{X}-100,0.1 \%$ 2-mercaptoethanol, with protease inhibitors (leupeptin, pepstatin A, antipain, aprotinin, and chymostatin, each at $20 \mu \mathrm{g} / \mathrm{ml}$, and $1 \mathrm{~mm}$ PMSF). Samples were analyzed by SDSPAGE and immunoblotting with anti-GFP, anti-pY, and antiGST (Pharmacia) antibodies.

\section{Acknowledgments}

We thank Janel Warmka, Scott Spencer, Mike Edwards, and Nick Tolwinski for technical support, Mike Gustin, Sean O'Rourke, Ira Herskowitz, Jean Hirsch, and Charles Zuker for reagents, and Anne Burkholder, Chris Arkind, Tim Lewis, Natalie Ahn, and Paul Shapiro for critically reading this manuscript. This work was supported by grants from the National Science Foundation (NSF), the March of Dimes, and an National Institutes of Health (NIH) Training Grant (GM07135).

The publication costs of this article were defrayed in part by payment of page charges. This article must therefore be hereby marked "advertisement" in accordance with 18 USC section 1734 solely to indicate this fact.

\section{References}

Ben-Levy, R., S. Hooper, R. Wilson, H.F. Paterson, and C. J. Marshall. 1998. Nuclear export of the stress-activated protein kinase p38 mediated by its substrate MAPKAP kinase-2. Curr. Biol. 8: 1049-1057.

Biggs, W.H., K.H. Zavitz, B. Dickson, A. van der Straten, D. Brunner, E. Hafen, and S.L. Zipursky. 1994. The Drosophila rolled locus encodes a MAP kinase required in the sevenless signal transduction pathway. EMBO J. 13: 1628-1635. 
Bott, C.M., S.G. Thorneycroft, and C.J. Marshall. 1994. The sevenmaker gain-of-function mutation in $\mathrm{p} 42 \mathrm{MAP}$ kinase leads to enhanced signaling and reduced sensitivity to dual specificity phosphatase action. FEBS Lett. 352: 201-205.

Brewster, J.L., T. de Valoir, N.D. Dwyer, E. Winter, and M.C. Gustin. 1993. An osmosensing signal transduction pathway in yeast. Science 259: 1760-1763.

Brunet, A., D. Roux, P. Lenormand, S. Dowd, S. Keyse, and J. Pouyssegur. 1999. Nuclear translocation of $\mathrm{p} 42 / \mathrm{p} 44$ mitogen-activated protein kinase is required for growth factorinduced gene expression and cell cycle entry. EMBO $\mathrm{J}$. 18: 664-674.

Brunner, D., N. Oellers, J. Szabad, W.H. Biggs, S.L. Zipursky, and E. Hafen. 1994. A gain-of-function mutation in Drosophila MAP kinase activates multiple receptor tyrosine kinase signaling pathways. Cell 76: 875-888.

Chen, R.H., C. Sarnecki, and J. Blenis. 1992. Nuclear localization and regulation of erk- and rsk-encoded protein kinases. Mol. Cell. Biol. 12: 915-927.

Ferrigno, P., F. Posas, D. Koepp, H. Saito, and P.A. Silver. 1998. Regulated nucleo/cytoplasmic exchange of HOG1 MAPK requires the importin beta homologs NMD5 and XPO1. EMBO I. 17: 5606-5614.

Fukuda, M., Y. Gotoh, and E. Nishida. 1997. Interaction of MAP kinase with MAP kinase kinase: Its possible role in the control of nucleocytoplasmic transport of MAP kinase. EMBO $\mathrm{J}$. 16: 1901-1908.

Gaits, F. and Russell P. 1999. Active nucleocytoplasmic shuttling required for function and regulation of stress-activated kinase Spc1/StyI in fission yeast. Mol. Biol. Cell 10: 13951407.

Gaits, F., G. Degols, K. Shiozaki, and P. Russell. 1998. Phosphorylation and association with the transcription factor Atf1 regulate localization of Spc1/Styl stress-activated kinase in fission yeast. Genes \& Dev. 12: 1464-1473.

Gietz, R.D. and A. Sugino. 1988. New yeast-Escherichia coli shuttle vectors constructed with in vitro mutagenized yeast genes lacking six-base pair restriction sites. Gene 74: 527534.

Gonzalez, F.A., A. Seth, D.L. Raden, D.S. Bowman, F.S. Fay, and R.J. Davis. 1993. Serum-induced translocation of mitogenactivated protein kinase to the cell surface ruffling membrane and the nucleus. J. Cell Biol. 122: 1089-1101.

Guthrie, C. and R.G. Fink. 1991. Guide to yeast genetics and molecular biology. Methods Enzymol. 194.

Hovland, P., J. Flick, M. Johnston, and R.A. Sclafani. 1989. Galactose as a gratuitous inducer of GAL gene expression in yeasts growing on glucose. Gene 83: 57-64.

Han, J., Y. Jiang, Z. Li, V. Kravchenko, and R.J. Ulevitch. 1997. Activation of the transcription factor MEF2C by the MAP kinase p38 in inflammation. Nature 386: 296-299.

Jacoby, T., H. Flanagan, A. Faykin, A.G. Seto, C. Mattison, and I. Ota. 1997. Two protein-tyrosine phosphatases inactivate the osmotic stress response pathway in yeast by targeting the mitogen-activated protein kinase, Hog1. J. Biol. Chem.. 272: 17749-17755.

Khokhlatchev, A.V., B. Canagarajah, J. Wilsbacher, M. Robinson, M. Atkinson, E. Goldsmith, and M.H. Cobb. 1998. Phosphorylation of the MAP kinase ERK2 promotes its homodimerization and nuclear translocation. Cell 93: 605-615.

Lenormand, P., C. Sardet, G. Pages, G. L'Allemain, A. Brunet, and J. Pouyssegur. 1993. Growth factors induce nuclear translocation of MAP kinases (p42mapk and p44mapk) but not of their activator MAP kinase kinase (p45mapkk) in fibroblasts. J. Cell Biol. 122: 1079-1088.
Lenormand, P., J.M. Brondello, A. Brunet, and J. Pouyssegur. 1998. Growth factor-induced p42/p44 MAPK nuclear translocation and retention requires both MAPK activation and neosynthesis of nuclear anchoring proteins. I. Cell Biol. 142: 625-633.

Maeda, T., A.Y. Tsai, and H. Saito. 1993. Mutations in a protein tyrosine phosphatase gene (PTP2) and a protein serine/threonine phosphatase gene (PTC1) cause a synthetic growth defect in Saccharomyces cerevisiae. Mol. Cell. Biol. 13: 54085417.

Maeda, T., S.M. Wurgler-Murphy, and H. Saito. 1994. A twocomponent system that regulates an osmosensing MAP kinase cascade in yeast. Nature 369: 242-245.

Mattison, C.P., S.S. Spencer, K.A. Kresge, J. Lee, and I.M. Ota. 1999. Differential Regulation of the cell wall integrity pathway in budding yeast by the protein tyrosine phosphatases, Ptp2 and Ptp3. Mol. Cell. Biol. 19: 7651-7660.

O'Rourke, S.M. and I. Herskowitz. 1998. The Hog1 MAPK prevents cross talk between the HOG and pheromone response MAPK pathways in Saccharomyces cerevisiae. Genes \& Dev. 12: 2874-2886.

Ota, I.M. and A. Varshavsky. 1992. A gene encoding a putative protein tyrosine phosphatase suppresses lethality of an $\mathrm{N}$ end rule-dependent mutant. Proc. Nat1. Acad. Sci. 89: 23552359.

Reiser, V., H. Ruis, and G. Ammerer. 1999. Kinase activitydependent nuclear export opposes stress-induced nuclear accumulation and retention of Hog 1 mitogen-activated protein kinase in the budding yeast Saccharomyces cerevisiae. Mol. Biol. Cell 10: 1147-1161.

Schuller, C., J.L. Brewster, M.R. Alexander, M.C. Gustin, and H. Ruis. 1994. The HOG pathway controls osmotic regulation of transcription via the stress response element (STRE) of the Saccharomyces cerevisiae CTT1 gene. EMBO I. 13: 43824389.

Traverse, S., N. Gomez, H. Paterson, C. Marshall, and P. Cohen. 1992. Sustained activation of the mitogen-activated protein (MAP) kinase cascade may be required for differentiation of PC12 cells. Comparison of the effects of nerve growth factor and epidermal growth factor. Biochem. I. 288: 351-355.

Watanabe, Y. and M. Yamamoto, 1996. Schizosaccharomyces pombe pcr1+ encodes a CREB/ATF protein involved in regulation of gene expression for sexual development. Mol. Cell. Biol. 16: 704-711.

Wurgler-Murphy, S.M., T. Maeda, E.A. Witten, and H. Saito. 1997. Regulation of the Saccharomyces cerevisiae HOG1 mitogen-activated protein kinase by the PTP2 and PTP 3 protein tyrosine phosphatases. Mol. Cell. Biol. 17: 1289-1297.

York, R.D., H. Yao, T. Dillon, C.L. Ellig, S.P. Eckert, E.W. McCleskey, and P.J. Stork. 1998. Rap1 mediates sustained MAP kinase activation induced by nerve growth factor. $\mathrm{Na}$ ture 392: 622-626.

Zhan, X.L., R.J. Deschenes, and K.L. Guan. 1997. Differential regulation of FUS3 MAP kinase by tyrosine-specific phosphatases PTP2/PTP3 and dual-specificity phosphatase MSG5 in Saccharomyces cerevisiae. Genes \& Dev. 11: 1690-1702.

Zhou, G., Z.Q. Bao, and J.E. Dixon. 1995. Components of a new human protein kinase signal transduction pathway. J. Biol. Chem. 270: 12665-12669.

Zuniga, A., J. Torres, J. Ubeda, and R. Pulido. 1999. Interaction of mitogen-activated protein kinases with the kinase interaction motif of the tyrosine phosphatase PTP-SL provides substrate specificity and retains ERK2 in the cytoplasm. I. Biol. Chem. 274: 21900-21907. 


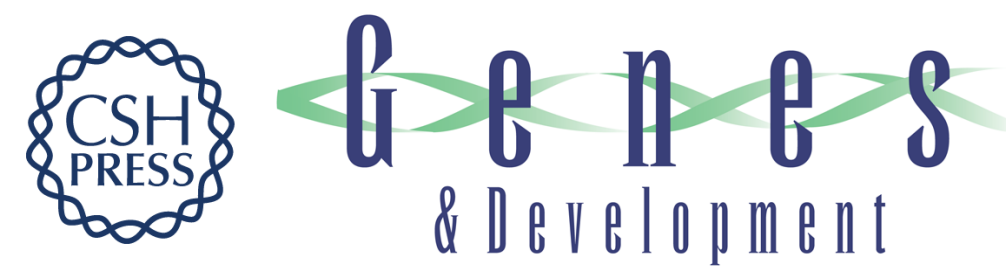

\section{Two protein tyrosine phosphatases, Ptp2 and Ptp3, modulate the subcellular localization of the Hog1 MAP kinase in yeast}

Christopher P. Mattison and Irene M. Ota

Genes Dev. 2000, 14:

Access the most recent version at doi:10.1101/gad.14.10.1229

References

This article cites 32 articles, 21 of which can be accessed free at: http://genesdev.cshlp.org/content/14/10/1229.full.html\#ref-list-1

License

Email Alerting

Receive free email alerts when new articles cite this article - sign up in the box at the top Service right corner of the article or click here.

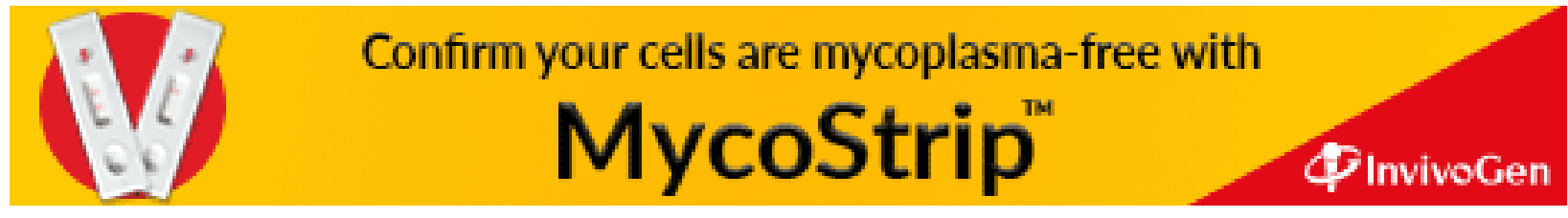

